\title{
Euthanasia in Wildlife Rehabilitation
}

\author{
DAVE MCRUER, MSC, DVM, DACVPM \\ PARKS CANADA \\ Charlottetown, Prince Edward Island, Canada
}

\begin{abstract}
Euthanasia is the act of humanely inducing death in an animal with minimal pain and distress (AVMA 2013). While the decision to euthanize a patient may be based on objective criteria, and the alleviation of suffering may be the ultimate and desired goal, euthanasia is one of the most difficult tasks faced in wildlife medicine. Wildlife caregivers place significant value on animal welfare. The decision to euthanize, therefore, should be given appropriate consideration and should focus on the animal's best interest. This article explores the ethics of animal euthanasia in wildlife rehabilitation and the methods commonly used to perform the task.
\end{abstract}

Keywords: Euthanasia, AVMA, wildlife, rehabilitation, five freedoms, inhalants, chemical, physical

\section{INTRODUCTION}

It is widely believed that animals, unlike humans, remain focused on the present and do not think about or anticipate their long-term future. Behaviors like migration, hibernation, dam building, and sitting by a water hole waiting for prey are likely the result of innate tendencies, learned behaviors, or a combination of both, rather than a conscious effort to plan for future events. It is accepted that animals have the ability to remember, and therefore events that happen 'now' may taint some or all of their previous experiences. This concept is summarized by Bernard Rollin, a philosopher of animal welfare and animal consciousness (2009). Rollin uses the example of a dog that has been treated lovingly, being beaten to death by his owner. In addition to the physical pain, the dog likely experiences confusion between all its past experiences and the treatment it is presently enduring. This negative experience suffered at the end of life may color the sum total of all of the animal's previous experiences. Therefore, events that happen at death may have special significance to animals, just like those

Dave McRuer, MSc, DVM, DACVPM is a Wildlife Health Specialist with Parks Canada and an adjunct faculty member at the Atlantic Veterinary College. Previously, he was the Director of Veterinary Services at the Wildlife Center of Virginia where he served for 11 years. Dave is a former National Wildlife Rehabilitators Association (NWRA) board member and former course coordinator for the NWRA veterinary student Wildlife Medicine Course. experienced by humans. Likewise, an animal that has spent its entire life in the wild may have all his previous experiences eclipsed by a painful or stressful death. Thus, it is important that those who euthanize wild animals do so in a manner that minimizes pain, stress, and anxiety.

The ultimate goal of wildlife rehabilitation is to return healthy individuals that have suffered injury or abandonment to their habitat so they may become contributing members of the population. Minimum Standards for Wildlife Rehabilitation (Miller 2012) includes a list of criteria that a patient must meet in order to be considered for release. Minimum standards for release of wildlife following rehabilitation include:

- Exhibit full recovery from the original injury or from injuries incurred while in care.

- Be no longer in need of medical care.

- Exhibit no signs of active disease.

- Have normal laboratory values, if tested (PCV, TS, BUN, etc.).

- Possess pelage or plumage that is adequate for that species to survive.

- Possess adequate vision to find/catch food and maneuver in a normal manner.

- Exhibit locomotive skills necessary for that species to survive.

- Demonstrate the fight or flight behavioral response.

- Demonstrate proper foraging behavior (self-feeding if raised in captivity).

- Demonstrate proper species behavior (not improperly imprinted).

- Be of correct age for independent survival.

- Be of correct weight for that sex, species, age, and season.

- Exhibit waterproof pelage/plumage sufficient for that species. 
An animal that cannot be released may be considered for placement in permanent captivity if it is believed the animal can have an adequate quality of life based on both biological and non-biological factors. For example, a docile juvenile red-tailed hawk (Buteo jamaicensis) with bilateral retinal injuries may adjust and thrive in captivity, whereas an adult Cooper's hawk (Accipiter cooperii) may destroy itself in an aviary due to internal and external stresses that cause it to traumatize itself within the enclosure. Simply keeping an animal alive does not necessarily ensure an adequate quality of life. When considering permanent placement of a wild animal, it is appropriate to consider the Five Freedoms (FAWC 2009), a list created for the livestock industry and shown below, but equally applicable to any animal maintained in captivity.

1. Freedom from hunger and thirst. The animal should be given ready access to fresh water and a diet to maintain full health and vigor.

2. Freedom from discomfort. An appropriate environment, including shelter and a comfortable resting area, must be provided.

3. Freedom from pain, injury, or disease. Preventative care, rapid diagnosis, and medical treatment must be available.

4. Freedom to express normal behavior. The animal should have sufficient space, proper facilities, and the company of its own kind if appropriate.

5. Freedom from fear and distress. Conditions and treatment that cause mental suffering should be avoided.

If an animal cannot be released and the five freedoms are not possible in captivity, euthanasia may be the only way a rehabilitator can ensure freedom from suffering.

The foundation for appropriate methods of euthanasia has been addressed in a series of updated reports by the American Veterinary Medical Association (AVMA, formally the AVMA Panel for Euthanasia), the last of which was published in 2013. These publications focus mostly on domestic animals and provide limited information on laboratory and nondomestic species. In order to address euthanasia in a broader range of nondomestic species and free-ranging wildlife, the professional zoological and wildlife medicine community published Guidelines for Euthanasia in Nondomestic Animals (Baer 2006). While not as broadly circulated or as widely accepted as the AVMA Guidelines for the Euthanasia of Animals (AVMA 2013), this report offers additional information that is at least as relevant to wildlife rehabilitators. Other professional organizations, including the American Association of Mammalogists (Sikes 2011) and the American College of Laboratory Animal Medicine (ACLAM 2005), also publish guidelines for euthanizing nondomestic species. Most of these documents use the AVMA guidelines as their foundation, and expand their guidance in areas that are underreported. Wildlife rehabilitators should be aware of the differences between these documents. It is up to individuals to seek training in what they feel is the most humane form of euthanasia that is both legal and within their practical limits.

Minimum Standards for Wildlife Rehabilitation (Miller 2012) provides information on the various euthanasia agents, but does not specify which agent should be used in which situation, as the circumstances may change based on species, life stage, physiological state, resources, location, and so on. Instead, it lists the following criteria for the appropriate method of euthanasia:

- Produces rapid loss of consciousness and death.

- Exhibits consistent and predictable action.

- Is easily and safely administered by properly trained personnel.

- Causes minimal psychological stress to the animal. Ways to minimize stress prior to euthanasia include minimizing exposure to humans and other predator species, stress vocalizations from other animals, and blood, which may carry odors or pheromones that result in anxiety. Effective, proper restraint also minimizes stress.

- Is not subject to abuse by humans.

- Interrupts consciousness and reflexes simultaneously. Some drugs, such as depolarizing and nondepolarizing muscle relaxants, strychnine, nicotine, and magnesium salts, induce muscle paralysis without loss of consciousness and should not be used as the primary method of euthanasia, since the animal still can perceive what is happening.

- Is not a sanitation or environmental problem.

- Results in no tissue changes that would affect a postmortem diagnosis. For example, the brain should not be destroyed in a rabies vector species needed for testing.

- Is economical and readily available.

- Causes minimal emotional effects to observers and participants.

It is a paradox that those who desire to help injured animals and who are involved with wildlife rehabilitation may ultimately end up causing the death of countless patients through euthanasia. Even when the technique is appropriate, pain and distress 
is minimized, and the animal's suffering is relieved in a peaceful and humane manner, those who must perform euthanasia often experience physical and psychological stress. Termed 'moral stress' or 'compassion fatigue,' this stress serves as a reminder of the responsibility associated with euthanasia. When performing euthanasia fails to evoke an emotional response in a caregiver, it may be prudent for the caregiver to take a break and reflect upon the significance of the procedure. It is not a bad thing if a caregiver cannot personally euthanize an animal; however, an alternative plan to end the life of a suffering patient should be in place.

All those involved in wildlife rehabilitation should have an established plan for humanely euthanizing permanently injured or suffering wild patients. When appropriate, attempts should be made to anesthetize or sedate patients prior to euthanasia in order to render the animal unconscious and minimize distress. In most states and provinces, wildlife rehabilitators need to have a relationship with a veterinarian to acquire prescription medications for their patients. For veterinarians with scheduled drug licenses, sodium pentobarbital is the gold standard for euthanizing all classes of wildlife. This drug is strictly regulated and in most areas must be prescribed and administered by a veterinarian. The most common way that wildlife rehabilitators can facilitate the humane euthanasia of injured wildlife is to establish a working relationship with their local veterinarian. In some cases injured animals can be taken to the veterinarian's clinic, while in other situations the veterinarian may come to the wildlife care center on a regular schedule. Ideally, a veterinarian should always be available to euthanize an injured patient in need. However, alternative means of euthanasia often must be sought.

Historically, the inhalant anesthetic gas halothane commonly was used in chambers to euthanize small wildlife patients. However, this drug is no longer manufactured in the United States. While carbon dioxide chambers can be used to euthanasia small animals, ethical dilemmas have arisen with its use in recent years (see "Methods of Euthanasia," below). Physical methods of euthanasia also may be used; however, they may be visually and emotionally displeasing, both to the operator and to observers. Regardless of the chosen method, all euthanasia techniques should be discussed with a veterinarian or trained professional in order to provide proper training and ensure the most humane form of euthanasia possible is used under the circumstances.

Personal safety and sanitation should be considered carefully before performing euthanasia. Many of the accepted methods of euthanasia may cause harm to the operator; thus, appropriate safety precautions should be given as much attention as the euthanasia procedure itself. Appropriate training and licensure, where applicable, should be obtained prior to using firearms to euthanize animals. Chambers used to euthanize animals with carbon dioxide or anesthetic gases should be properly scavenged (removed) to protect the operator and bystanders from secondary exposure. In humans, anesthetic gases may result in dizziness and headaches. Additionally, long-term exposure is associated with adverse effects on liver, kidney, and other organ function, and short-term exposure during early pregnancy has resulted in spontaneous abortions and congenital abnormalities. Barbiturates used in animal euthanasia such as sodium pentobarbital are regulated strictly, as they may be abused or adversely affect human health if accidentally administered. In addition, barbiturates produce secondary toxicosis in animals unfortunate enough to ingest an improperly disposed euthanized animal. It is important that all animals euthanized by chemical means be properly disposed.

As mentioned, one of the key elements in a humane euthanasia is the alleviation of pain. Pain is defined as the sensation that results when nerve impulses from peripheral pain receptors, or nociceptors, reach a functioning cerebral cortex and associated subcortical brain structures (Thurmon 1996). When a noxious stimulus activates nociceptors, impulses travel up nerve pathways to the spinal cord and brain. Stimulation in the spinal cord creates reflexes, such as the withdrawal of the limb from the noxious stimulus, while stimulation within the brain of a conscious animal produces the perception of pain. Pain may be alleviated by drugs that prevent the stimulation of the nociceptors by stopping or decreasing the impulses at their source, which prevents the transmission of impulses from the nociceptors to the brain, or which decreases the cerebral cortex activity, such as when an animal is rendered unconscious. If the brain cannot receive or process nerve impulses from noxious stimuli, pain cannot be perceived.

It also should be noted that most of our knowledge of pain comes from mammals and birds. Fish, amphibians, and reptiles lack anatomical features associated with the pain pathway found in the studied classes, and therefore it is unknown if these species feel pain in the same manner. Regardless, it is ethically responsible to assume that these animals do feel pain (Meyer and Morgan 2006). In all wildlife euthanasia procedures, every effort should be made to control pain. 
All methods of euthanasia cause death by one of three basic mechanisms: (1) hypoxia (inadequate oxygen supply to the cells and tissues of the body); (2) direct depression of neurons (brain nerve cells) necessary for life function; and, (3) physical disruption of brain activity (AVMA 2013).

The most common form of hypoxia occurs when an animal is deprived of oxygen, such as when carbon dioxide displaces oxygen in induction chambers. This method is commonly used by wildlife rehabilitators for small mammals and birds. Less common forms of creating hypoxia include the application of carbon monoxide, which prevents the uptake of oxygen by red blood cells, and exsanguination, or blood loss, until death results. The use of carbon monoxide is universally discouraged without the proper equipment, as concentrations over 10 percent are explosive, and the odorless gas presents an extreme safety risk to the operator and others. Exsanguination should be performed only on animals that are already unconscious, as it may cause severe anxiety otherwise.

The depression of the neurons results in the animal first losing consciousness and then succumbing to death. As the animal becomes unconscious, muscle contractions, reflexive struggling, and vocalization may occur due to the release of inhibitory neurons. While this may be disturbing for the operator and observers, these movements only occur after the animal has lost consciousness and cannot perceive pain. These uncoordinated movements and vocalizations are equivalent to the second stage of anesthesia. An example of a drug that depresses neurons is sodium pentobarbital, the euthanasia drug of choice for most veterinarians.

Physical disruption of brain activity requires a more personal interaction between the animal and the operator and may not be the ideal choice for all wildlife rehabilitators. The process usually involves an initial concussive stunning blow to the skull followed by direct destruction of the brain tissue. The latter is performed with a penetrating captive bolt, a bullet, or a pithing probe. Once the midbrain is destroyed successfully, respiratory and cardiac arrest quickly follow. The operator must be highly skilled, as failure to ensure a quick and painless death may lead to significant suffering.

The ideal method of euthanasia should result in the rapid loss of consciousness, should minimize pain and distress, and is followed by respiratory and cardiac arrest (AVMA 2013). Prior to the loss of consciousness, all efforts should be taken to minimize patient distress and anxiety. Euthanasia should be conducted only by those trained in a given technique, with experience restraining the animal being euthanized, adequate knowledge of the animal's behavior both prior to and following restraint, and appropriate state and federal authorization. Information on euthanasia techniques is available through a variety of rehabilitation and non-rehabilitation resources. However, technical advice from professionals such as veterinarians, research laboratories, experienced wildlife rehabilitators, and biologists trained in field protocols should be sought before performing euthanasia without supervision. It also is important to remember that a follow-up examination always is required to ensure the animal is dead. In addition to absence of a heartbeat, there should be no reaction to a hard toe pinch and the pupils should be fixed, dilated, and unresponsive to the touching of the cornea. Respiratory arrest is not enough to ensure that an animal is dead.

\section{EuthanAsia Methods}

Methods of euthanasia can be divided into three categories-inhalant, chemical, and physical. The following list of methods is not exhaustive, but covers the most commonly recommended, conditionally acceptable, and unacceptable forms of euthanasia in animals (AVMA 2013; Baer 2006; Miller 2012). Even if recommended, it is unethical to perform these techniques without the proper training and licenses/ permits where they apply. Those charged with the responsibility of euthanasia should always seek to learn the methods they feel are most humane, and try to make improvements on techniques with the goal of decreasing pain and distress in the animal.

Inhalants. Inhalants are vapors and gases that exert their effect through the respiratory system. In general, gaseous inhalants are ill-suited for animals resistant to higher levels of hypoxia (such as turtles, crocodilians, amphibians, bats, neonatal animals, and diving birds and mammals), as induction into loss of consciousness can be prolonged significantly. Although MS-222 and benzocaine hydrochloride are not gaseous agents, they have been included in the inhalant section because they act through an amphibian's respiratory system.

Anesthetic Gases-Many of the anesthetic inhalants used to induce and maintain anesthesia in animals can be overdosed intentionally for euthanasia. These agents vary in their induction characteristics, but in general they have the ability to rapidly induce unconsciousness before causing death from hypoxia through respiratory depression. The AVMA recommends the followings inhalants in order of preference: isoflurane, halothane, sevoflurane, enflurane. The most commonly used inhalant anesthesia, isoflurane, is considered the best choice, but its pungent nature 
Table 1. Inhalant Methods of Euthanasia.

\begin{tabular}{|c|c|}
\hline \multicolumn{2}{|c|}{ Inhalant Methods } \\
\hline \multicolumn{2}{|c|}{ Anesthetic gases: halothane, enflurane, isoflurane, sevoflurane } \\
\hline Species & Amphibians, reptiles, birds, mammals \\
\hline Status & $\begin{array}{l}\text { Conditionally acceptable; acceptable in small animals }<7 \mathrm{~kg}(15 \mathrm{lb}) \text {; should be followed by } \\
\text { other recommended methods to ensure death. }\end{array}$ \\
\hline \multicolumn{2}{|c|}{ Carbon dioxide $\left(\mathrm{CO}_{2}\right)$} \\
\hline Species & Terrestrial reptiles, birds, mammals \\
\hline Status & $\begin{array}{l}\text { Conditionally acceptable-gradual fill recommended; bats, diving animals, and young animals } \\
\text { may be more resistant. }\end{array}$ \\
\hline \multicolumn{2}{|c|}{ Carbon monoxide (CO) } \\
\hline Species & Reptiles, birds, mammals \\
\hline Status & $\begin{array}{l}\text { Conditionally acceptable IF all safety and specialized equipment conditions are met. } \\
\text { Generally NOT recommended for use in wildlife rehabilitation. }\end{array}$ \\
\hline \multicolumn{2}{|c|}{ Ether and Chloroform } \\
\hline Species & None \\
\hline Status & Unacceptable \\
\hline \multicolumn{2}{|c|}{ Nitrous oxide } \\
\hline Species & Amphibians, reptiles, birds, mammals \\
\hline Status & Unacceptable as a sole means of euthanasia. \\
\hline \multicolumn{2}{|c|}{ MS-222 (tricaine methane sulfonate) } \\
\hline Species & Amphibians \\
\hline Status & Recommended \\
\hline \multicolumn{2}{|c|}{ Benzocaine hydrochloride } \\
\hline Species & Amphibians \\
\hline Status & Recommended \\
\hline
\end{tabular}

can lead many animals to hold their breath and thus increase induction times. While halothane was once commonly used in wildlife rehabilitation, it is no longer manufactured in the United States. Sevoflurane, while less potent than isoflurane, is not nearly as malodorous and thus can facilitate more rapid induction.

There are two common methods of administering these agents to animals. The inhalant can be run through an anesthetic vaporizer and delivered via a mask or flow into a small induction chamber. A quicker alternative is to place a gauze or cotton ball moistened with the agent into an induction chamber with the animal. It is important to physically separate the animal from the source of the vapors, as direct contact can be irritating. The chamber should be large enough for the animal to turn around but not too large, as this prolongs the conscious phase of the procedure and generates excess waste gas.

Although these agents can be used as the sole means of euthanasia, they most often are used as an anesthetic to allow easier, stress-free handling for the application of a secondary method of euthanasia. If the inhalant agent is the sole method of euthanasia, the animal should be kept in the chamber for at least 15 minutes after the last visible breath. As always, death needs to be confirmed with a thorough evaluation for a heartbeat, pulse, or any other sign of life.
Many sources recommend follow-up methods in all animals, but especially in reptiles (Woodbury 2014; Mader 2006).

There are a number of health concerns for humans who use inhalant gases to perform euthanasia. Exposure to isoflurane has been shown to be deleterious to developing babies, so pregnant women should not be exposed. Chronic exposure to other inhalants may carry health concerns. For these reasons, scavenging devices should be used to prevent human exposure to waste gases. At the very least, chambers containing inhalant gases should be opened in well ventilated areas.

Induction with these agents can put an animal through an excitement phase. Although the animal can no longer perceive what is happening, it may have involuntary muscle spasms and vocalization. These drugs may not work well in animals with respiratory diseases or in diving animals that are used to holding their breath. Lastly, these agents leave residues in the euthanized animal, so carcasses must be disposed of appropriately to prevent secondary toxicosis in other species that may consume the remains.

Carbon Dioxide $\left(\mathrm{CO}_{2}\right)$-Carbon dioxide long has been a staple of euthanasia for wildlife and agricultural, shelter, and lab animals. Its many advantages include a high level of safety to personnel (relatively 
nontoxic, nonflammable, and nonexplosive), the commercial availability of cylinders and chambers for use in euthanasia, and the absence of residues in the carcasses. It is relatively inexpensive and can be used to euthanize large numbers of animals with considerably less labor than individual methods like sodium pentobarbital injections. The AVMA has approved $\mathrm{CO}_{2}$ in most species, with the exception of marine mammals, aquatic reptiles, ruminants, and horses. The Canadian Council for Animal Care guidelines also suggest that carbon dioxide not be used in amphibians and fish due to the formation of carbonic acid when $\mathrm{CO}_{2}$ dissolves in water. State and provincial use for euthanasia may vary, so it is important to investigate local laws before implementing this method.

In recent years, welfare issues have been raised concerning the use of $\mathrm{CO}_{2}$. Many studies have demonstrated that the $\mathrm{CO}_{2}$ concentrations needed to rapidly render animals unconscious (in a minute or less) also are likely associated with pain and discomfort (Neil and Weary 2006; Tidemann and King 2009; Makowska et al 2009; Leach et al 2004). This continues to be a controversial topic, with some sources recommending the use of $\mathrm{CO}_{2}$ only after general anesthesia already has been administered.

Carbon dioxide works by sinking to the bottom of the chamber and displacing ambient air. The lack of oxygen around the patient causes direct depression of the central nervous system, followed by cardiac and respiratory arrest. The literature describes both prefilled and gradually filled $\mathrm{CO}_{2}$ chambers. Both of these methods have been associated with pain and distress in the patient, originating from difficulty in breathing and the production of carbonic acid (formed from $\mathrm{CO}_{2}$ and water) on the mucous membranes of the eyes and respiratory tract. Recent studies have found that gradual fill protocols are desirable over prefilled protocols as the latter may cause pain prior to unconsciousness in the animal. Current recommendations are for the animal to be placed in a chamber that is then gradually filled with $\mathrm{CO}_{2}$ at a rate of 30 percent of the chamber volume per minute. Low flow volume per minute displacement rate (10\%) tends to cause increased panic and high volume per minute displacement rate (100\%) causes pain and anxiety (Hickman et al 2016). It is not recommended to dilute $\mathrm{CO}_{2}$ with oxygen, as this is more distressing and results in a delayed death. After the onset of respiratory arrest, the animal should be left in the closed chamber for at least one minute and up to fifteen, to ensure that the animal does not revive.

$\mathrm{CO}_{2}$ concentrations necessary for humane euthanasia vary considerably and need to be higher in certain species, such as burrowing animals, reptiles, amphibians, bats, and diving animals, and in young animals (hatchlings, neonates), as these may be more resistant to $\mathrm{CO}_{2}$. Only compressed $\mathrm{CO}_{2}$ from cylinders should be used for euthanasia; gas from dry ice or a chemical reaction is not considered appropriate.

Carbon Monoxide (CO)-Carbon monoxide is a potent metabolic poison that tightly binds to the hemoglobin in the blood to create carboxyhemoglobin. This can lead rapidly to hypoxia, as carboxyhemoglobin is unable to bind to and distribute oxygen throughout the body. Acute exposure to the gas can cause headaches, vomiting, nausea, weakness, loss of consciousness, and death. Since CO is a cumulative poison, small exposures can add up over hours. Chronic exposure has been linked to heart disease and birth defects. The gas is clear, odorless, and difficult to detect by methods other than expensive $\mathrm{CO}$ detectors. At a concentration of greater than 10 percent, the gas also is potentially explosive. All of these factors make using carbon monoxide very dangerous to personnel performing euthanasia. As a result, the AVMA recommends that this method only be used by well-trained individuals taking the proper precautions, including the use of a well-ventilated outside area with explosion-proof equipment. Only compressed CO cylinders are considered an appropriate source; chemical reactions and exhaust from combustion engines (tail pipes), which cause distress and pain, are inappropriate.

Ether and Chloroform-Both drugs cause direct depression of the central nervous system and usually are administered in an induction chamber. Ether's explosive nature can make its use dangerous. Additionally, it irritates exposed mucous membranes. Chloroform is a known hepatotoxin that presents too great a danger to personnel to serve as a viable euthanasia agent.

Nitrous Oxide-Nitrous oxide is an inhalant anesthetic used both in humans and as an adjunct anesthetic gas in animals. It is not potent enough to induce anesthesia in animals if administered on its own, but can displace enough oxygen to create hypoxemia before respiratory and cardiac arrest. For this reason, nitrous oxide is never acceptable as the sole agent of euthanasia in animals.

MS-222 (Tricaine Methane Sulfonate)-Because of their small size, soaking amphibians in an MS-222 water bath can be an effective method of inducing anesthesia and euthanasia. The drug works similarly to inhalant anesthetic agents, first depressing the central nervous system and then causing respiratory and cardiac arrest. 
Since it is a concentrated stock solution, MS-222 can be diluted and administered for individual euthanasia. At concentrations greater than $500 \mathrm{mg} / \mathrm{L}$, the solution is acidic and needs to be buffered with sodium bicarbonate to reach a $\mathrm{pH}$ of 7.0 to 7.5. At this $\mathrm{pH}$, the solution is no longer irritating to the animal being euthanized. The amphibian should be kept in the water bath for at least fifteen minutes after the last breath is noted, at which time a physical method of euthanasia (e.g. decapitation, pithing, or exsanguination) should be performed to ensure death.

For safety, the stock solution ideally should be prepared under a fume hood, and gloves should be worn when handling the patient. The remaining agent can be disposed of with excess water in a drain that empties into a sewage system, but never into surface water. This agent leaves a residue in the carcass, so the body needs to be incinerated or properly disposed of to prevent secondary toxicosis in other wildlife species (Brown 2010).

Benzocaine Hydrochloride-Benzocaine hydrochloride has properties similar to MS-222. Generally, only a solution of $250 \mathrm{mg} / \mathrm{L}$ is considered adequate for euthanasia of amphibians. An application of 186 $\mathrm{mg} / \mathrm{kg}$ of 20 percent benzocaine gel, available as Orajel $^{\mathrm{TM}}$ (Church \& Dwight Co., Inc., Ewing, NJ), is also an effective euthanasia method, and is cheaper than MS-222 (Torreilles et al 2009). A secondary form of euthanasia should follow this method to ensure death.

Chemical. Injectable euthanasia agents, especially sodium pentobarbital, commonly are used for euthanasia in veterinary medicine. In most wildlife patients, intravenous injection (IV) is used only after general anesthesia has been induced, due to the difficulty and danger in attempting restraint for venous access. Even when physical restraint is attainable, it likely causes a high degree of stress to the animal. Venous access varies greatly among wildlife species, and it is the responsibility of the personnel to investigate the best locations for each species prior to administering an IV euthanasia drug. If IV access cannot be achieved in an energetic patient, a sedative or general anesthetic should be used first. Intravenous access may not be possible in very small animals like rodents and neonates. In these cases, intraperitoneal (into the abdomen) or intracardiac (into the heart) injection may be necessary in the sedated or anesthetized patient. In lizards, it is possible to perform an intracranial (into the brain) injection by inserting a needle through the parietal eye, under anesthesia (Mader 2006). Sedatives such as xylazine, ketamine, medetomidine, and tiletamine $\mathrm{HCL} /$ zolazepam HCL combination $\left(\right.$ Telazol $^{\circledR}$, Zoetis, Kalamazoo, MI) may be given as subcutaneous or intramuscular injections prior to euthanasia or delivered at three to five times the anesthetic dose for primary euthanasia. Doses for primary euthanasia using sedatives may change based on species. Recommended chemical euthanasia agents usually are administered as intravenous or intracardiac injections.

Sodium Pentobarbital-This agent is tightly regulated and only accessible through licensed veterinarians. Intravenous injection of sodium pentobarbital is used widely in small animal, exotic, and large animal medicine. Whenever possible, sodium pentobarbital should be administered intravenously, following sedation or the induction of a general anesthesia to decrease patient distress. Although the speed of action depends on the dosage, patient, and the rate and route of administration, barbiturates are fast-acting agents that initially depress consciousness before depressing the respiratory center and causing cardiac arrest. Sodium pentobarbital's stability in solution, low cost, potency, and prolonged duration of action make it the preferred barbiturate. Since barbiturates leave residues in the carcass, all animals euthanized with these agents should be incinerated or properly disposed of to prevent secondary toxicosis in other wildlife species.

Potassium Chloride-The use of potassium chloride in an animal that is neither sedated nor anesthetized is strictly prohibited, as it interferes with nerve conduction and cardiac muscle contraction, leading to cardiac arrest without affecting consciousness. This drug must be given by intracardiac or intravenous injection, and only should be administered to animals in a deep plane of anesthesia. Personnel administering this drug should be aware it may cause clonic spasms and rippling of muscle tissue. Potassium chloride has the advantage of being inexpensive, easy to store, and easily accessible, as it is not a controlled substance in the United States. It also does not appear to leave behind any residue, making it the injectable agent of choice for euthanasia of animals whose carcasses cannot or will not be disposed.

T-61 (Nonbarbiturate, nonnarcotic mixture of embutramide, mebozonium [mebenzonium] iodide, and tetracaine hydrochloride)-The embutramide in T-61 causes narcosis and respiratory depression, while the mebozonium simultaneously induces nondepolarizing muscular paralysis. This drug is not available in the United States, but is available in Canada and other countries. Concern has been raised that the paralytic effect may take effect prior to loss of 
Table 2. Chemical Methods of Euthanasia.

\begin{tabular}{|l|l|}
\hline \multicolumn{2}{|l|}{ Chemical Methods } \\
\hline Sodium pentobarbital \\
\hline Species & Amphibians, reptiles, birds, mammals \\
\hline Status & Recommended, considered the gold standard for animal euthanasia, license required. \\
\hline Potassium chloride \\
\hline Species & Amphibians, reptiles, birds, mammals \\
\hline Status & Conditionally acceptable; should only be used when animal is in a deep plane of anesthesia. \\
\hline $\begin{array}{l}\text { T-61 (nonbarbiturate, nonnarcotic mixture of embutramide, mebozonium [mebenzonium] iodide, and } \\
\text { tetracaine hydrochloride) }\end{array}$ \\
\hline Species & Amphibians, reptiles, birds, mammals \\
\hline Status & Acceptable if administered by intravenous injection by trained personnel. \\
\hline Neuromuscular blockers (succinylcholine) \\
\hline Species & None \\
\hline Status & $\begin{array}{l}\text { Unacceptable, unless the animal is in a deep plane of anesthesia, or followed immediately by } \\
\text { an accepted means of euthanasia prior to patient distress and death from asphyxiation. }\end{array}$ \\
\hline Formalin, ethanol, formaldehyde \\
\hline Species & None \\
\hline Status & Unacceptable \\
\hline Strychnine & \\
\hline Species & None \\
\hline Status & Unacceptable \\
\hline
\end{tabular}

consciousness, leading to distress (muscle activity and vocalization during injection) prior to death. Research on dogs and rabbits indicates that paralysis and unconsciousness occur simultaneously (Hellebrekers et al 1990). T-61 should be administered slowly by intravenous route to avoid dysphoria, which may cause distress in the people witnessing the process. The drug works quickly in dogs, cats, horses, birds, laboratory animals, and wildlife, but must be administered by intravenous injection by trained personnel.

Neuromuscular Blockers (Succinylcholine)Previously used in the capture and restraint of freeranging wildlife, succinylcholine is a potent depolarizing neuromuscular blocking agent that can be used to paralyze animals. Unfortunately, animals euthanized via this means suffocate slowly from respiratory paralysis without losing consciousness, making this class of drugs unacceptable as a sole means of euthanasia. If a neuromuscular blocker is used, a follow-up means of euthanasia must be administered quickly, before resulting asphyxiation causes pain and distress. When attempting to capture free-ranging wildlife, these circumstances are often unrealistic.

Formalin, Ethanol, Formaldehyde-Immersion in these chemicals is not an appropriate method of euthanasia unless deep levels of anesthesia have already been induced (ACLAM 2005). These methods occasionally may be needed to fix tissues in a laboratory situation but should not be used in wildlife rehabilitation.
Strychnine-Strychnine poisoning historically has been used to manage predator problems, but is no longer considered a valid method of euthanasia. Animals experiencing strychnine toxicity exhibit violent convulsions before expiring, and its use is considered inhumane.

Physical. Physical methods of euthanasia usually are performed after the animal has been anesthetized, as the second step of a two-step protocol. In the past, physical methods most often have been used when other methods are impractical or unavailable-in remote settings, when a large number of euthanasias need to be performed, with fractious patients. Some physical methods involve complex machinery that must be maintained to ensure it works quickly and correctly with each use. Other physical methods involve skilled techniques and should not be attempted on conscious animals until sufficient training has taken place. Physical methods that do not require pre-anesthesia have the advantage of producing a carcass that is free of chemical residues and therefore can be used as food. When rabies testing is required, methods that destroy the head or brain (gunshot, captive bolts, pithing, blows to the head, maceration) should not be used.

Gunshot-When a gun is used to perform euthanasia, the animal should be shot in the head with a caliber appropriate to destroy the brain at once. When performed correctly, this is considered humane 
Table 3. Physical Methods of Euthanasia.

\begin{tabular}{|c|c|}
\hline \multicolumn{2}{|c|}{ Physical Methods } \\
\hline \multicolumn{2}{|c|}{ Gunshot } \\
\hline Species & Amphibians, reptiles, birds, mammals \\
\hline Status & Conditionally acceptable; human safety and licensing concerns. \\
\hline \multicolumn{2}{|c|}{ Penetrating captive bolt } \\
\hline Species & Large species of reptiles and birds (alligators/ostriches), larger mammals \\
\hline Status & Conditionally acceptable \\
\hline \multicolumn{2}{|c|}{ Blunt force trauma to the head/stunning } \\
\hline Species & Amphibians, reptiles, birds, mammals \\
\hline Status & $\begin{array}{l}\text { Conditionally acceptable in small, thin-skulled animals when more direct and conventional } \\
\text { methods are unavailable. }\end{array}$ \\
\hline \multicolumn{2}{|l|}{ Pithing } \\
\hline Species & Amphibians, reptiles \\
\hline Status & $\begin{array}{l}\text { Conditionally acceptable; is commonly used as a secondary method of euthanasia in reptiles } \\
\text { following an accepted primary euthanasia protocol. }\end{array}$ \\
\hline \multicolumn{2}{|r|}{ _е } \\
\hline Species & Amphibians, reptiles, birds, small mammals \\
\hline Status & $\begin{array}{l}\text { Conditionally acceptable; should be followed by pithing in amphibians and reptiles; } \\
\text { appropriate for emergency euthanasia in remote locations. }\end{array}$ \\
\hline \multicolumn{2}{|c|}{ Cervical dislocation } \\
\hline Species & Reptiles, small birds, small mammals, all $<200 \mathrm{~g}$ \\
\hline Status & Conditionally acceptable; training required, prior sedation/general anesthesia is preferred. \\
\hline \multicolumn{2}{|c|}{ Electrocution } \\
\hline Species & None \\
\hline Status & Unacceptable without specialized equipment \\
\hline \multicolumn{2}{|c|}{ Exsanguination } \\
\hline Species & Amphibians, reptiles, birds, mammals \\
\hline Status & Conditionally acceptable; never acceptable on a conscious animal. \\
\hline \multicolumn{2}{|c|}{ Hypothermia/freezing } \\
\hline Species & None \\
\hline Status & Unacceptable \\
\hline \multicolumn{2}{|c|}{ Microwave } \\
\hline Species & Laboratory mice and rats \\
\hline Status & Unacceptable without specialized laboratory equipment. \\
\hline \multicolumn{2}{|l|}{ Burning } \\
\hline Species & None \\
\hline Status & Unacceptable \\
\hline \multicolumn{2}{|c|}{ Thoracic compression } \\
\hline Species & Small birds and mammals \\
\hline Status & $\begin{array}{l}\text { Conditionally acceptable following primary anesthesia. Cardiac compression alone might be } \\
\text { acceptable is small birds. }\end{array}$ \\
\hline \multicolumn{2}{|c|}{ Kill traps } \\
\hline Species & None \\
\hline Status & Unacceptable \\
\hline \multicolumn{2}{|c|}{ Drowning/smothering } \\
\hline Species & None \\
\hline Status & Unacceptable \\
\hline
\end{tabular}

euthanasia, as consciousness is lost immediately and death quickly follows. This form of euthanasia commonly occurs when first responders (police, animal control, etc.) dispatch animals, such as deer, that have been hit by vehicles. In these situations, a gunshot to the head is often more humane than having the animal endure the additional stress and pain of transportation to the nearest veterinary clinic. The other common use for gunshot euthanasia is for freeranging wildlife, where capture and restraint of the animal is impractical. In these cases, true euthanasia may not be possible, as a proper gunshot to the head is often very difficult to accomplish from a distance. A gunshot that causes massive damage to the heart and 
lungs is considered a humane killing in this situation and likely the best option available. When shotguns are used to shoot, the shot should be large enough to kill the animal outright.

When using a gun, the shooter needs to be trained in firearms safety and accurate marksmanship, and be knowledgeable in the landmarks required for humane euthanasia in the given species. Gunshot euthanasia is inherently dangerous and the path of the projectile needs to be considered to make sure surrounding people and animals are not injured. To increase safety, low-velocity ammunition, such as shotgun slugs, should be utilized. Gunshot euthanasia should be performed outdoors only, in an appropriate setting, far removed from public areas. The legality of using guns to perform euthanasia may vary from region to region, so local laws should be investigated before selecting this technique. As previously discussed, this method is not appropriate for animals suspected of having rabies, as it destroys the brain, which is used for viral testing.

Penetrating Captive Bolt-Much as with gunshot euthanasia, an accurately placed penetrating captive bolt can be a rapid sole method of euthanasia that causes significant trauma to the brain and renders the animal instantly unconscious. Penetration locations and power needed vary greatly by species, making it important to perform initial research to learn the correct protocols. Large animal captive bolt systems commonly are used for agricultural animals; some smaller animal versions have been used in laboratory animal medicine. This method has been used successfully in crocodilians (ACLAM 2005). Nonpenetrating captive bolt guns can be used successfully to stun an animal, but they are not appropriate as a sole method of euthanasia. Accepted follow-up methods, such as exsanguination, need to be used after nonpenetrating captive bolt guns. Similar to guns, this method is not appropriate for animals suspected of having rabies, as it destroys the brain, which is used for viral testing.

Blunt Force Trauma-The efficacy of a blow to the head is highly variable between species, making a follow-up form of acceptable euthanasia (e.g. exsanguination, pithing, decapitation) a necessity. Stunning may be especially effective in young neonates whose craniums are still soft and thin, and is used as the first part of a two-step euthanasia protocol in some reptiles. If this method is used, the operator must be skilled in its execution and aware that such methods often are aesthetically unpleasant. Because of the displeasing nature of this method, blunt force trauma should be replaced with alternate methods whenever possible.
Pithing-Pithing involves the destruction of the spinal cord and brainstem with a sharp instrument or rod. It most commonly is performed as a method of ensuring death in amphibians and reptiles after they have been overdosed with anesthetics or rendered unconscious through other means. Like other methods of euthanasia, training is required before attempting this method without supervision.

Decapitation-Decapitation is used primarily in small animals as a secondary method of euthanasia following a primary means of making the animal unconscious. This method may be used as a primary and/or sole method of euthanasia in birds and small mammals as an emergency procedure. Animals with a high tolerance for hypoxemia such as diving birds or mammals, bats, reptiles, amphibians, and neonatal animals either should be sedated or anesthetized beforehand or rapidly pithed afterward. Guillotines for rodents and small rabbits are available commercially, but most require extensive handling, likely to be very stressful to wildlife. Therefore, it is recommended that these animals first be anesthetized before decapitation. This method may be aesthetically displeasing. Those working with the guillotines also must be very careful to avoid injuring themselves. For some neonate mammals or very small reptiles and amphibians, a pair of sharp shears or even a knife may be appropriate for use in an unconscious animal.

Cervical Dislocation-Cervical dislocation is a specialized technique that rapidly separates the brain from the spinal cord, destroying the ascending sensory pathways for pain, and then depressing the central nervous system, causing respiratory and cardiac arrest. This is a method that should be practiced on cadavers or unconscious animals until the operator becomes proficient, and one that is limited to small animals. For most birds, cervical dislocation will result from rapid stretching of the neck. In rabbits, the head is held in one hand and the hind limbs in another. While the body is stretched, the head is hyperextended and twisted dorsally. In rodents less than 200 grams (6.43 ounces), the head is held between the thumb and forefinger while the body is pulled away (AVMA 2013). Operators should be aware that these methods may be aesthetically unpleasant.

Electrocution-Electrocution causes death from cardiac fibrillation. However, this does not occur instantaneously and does not first cause unconsciousness. It also is not recommended for animals weighing less than five kilograms (11 pounds), as circulatory failure and fibrillation do not always persist, leading to 'recoveries' from euthanasia. Euthanasia by this method is used in the poultry industry, which utilizes 
special equipment unavailable to the general public, but it is not an accepted method of euthanasia in wildlife species.

Exsanguination-It is known that hypovolemia can cause severe stress in animals, so although exsanguination is often used to ensure death, it should never be used as the sole method of euthanasia. When exsanguination is performed, the quickest and most efficient method is to cut through the large arteries and veins in the neck.

Hypothermia/Freezing-Using hypothermia as either a euthanasia method or a restraint technique is not considered humane and is strongly opposed by the AVMA. However, recent research in small ectotherms shows that whole-body cooling followed by freezing results in smooth brain activity declines with no perception of pain in cane toads (Rhinella marina) (Shine et al 2015; Lillywhite et al 2017). This method may become conditionally acceptable in small ectotherms in the future following additional research.

Microwave-In some research laboratories interested in brain metabolites, specialized microwave units are used for the euthanasia of mice and rats. These machines are considered humane, as they induce loss of consciousness and death in fractions of a second. However, it is important to note that these specialized units are not comparable to home microwaves. It is never appropriate to euthanize an animal with a home microwave unit.

Burning-It is never appropriate to euthanize an animal with either thermal or chemical burns. This technique is considered inhumane.

Thoracic Compression-Thoracic compression is a method of euthanasia historically used in small wild birds and mammals, performed by squeezing the animal's chest to prevent respiration. This method is currently not approved by the AVMA, wildlife rehabilitation community (Miller 2012), avian veterinarians, or zoological medical professionals due to concerns about its efficacy, prolonged duration of the procedure, potential for distress of the animal, and the perception of pain (Woodbury 2014; Harrison and Lightfoot 2005; Baer 2006). However, a recent paper comparing intraosseous pentobarbital administration versus thoracic compression in small song birds (house sparrows (Passer domesticus) and European starlings (Sturnus vulgaris) showed that cardiac compression (pressure directly over the heart rather than the entire thoracic cavity) might be an efficient method of euthanizing small birds (Paul-Murphy et al 2017). The AVMA does state this method of euthanasia is acceptable following initial anesthesia or on an animal that is already unconscious. This technique alone should never be attempted on small mammals, as their diaphragm continues to aid in respiration despite chest compression, leading to a prolonged and distressful death. Cervical dislocation, gunshot to the head, inhalant agents, or decapitation should be considered alternatives to this method in the field.

Kill Traps-Kill traps generally are considered unacceptable, as they do not always ensure a rapid and stress-free death consistent with the criteria for euthanasia. If animals in a remote area need to be euthanized, it is recommended that live traps be used for initial trapping, followed by an accepted euthanasia protocol. Kill traps also have the disadvantage that they can catch and kill the wrong species or individuals, leading to unintended deaths.

Drowning/Smothering-Neither drowning nor smothering is ever acceptable as a method of euthanasia. Both methods are considered cruel due to the painful and stressful nature of these deaths.

\section{LITERATURE CITED}

American College of Laboratory Animal Medicine. 2005. Report of the ACLAM Task Force on Rodent Euthanasia. American College of Laboratory Animal Medicine: Chester, NH. Accessed June 2016. <https://www.aclam.org/Content/files/ files/Public/Active/report_rodent_euth.pdf>.

American Veterinary Medical Association. 2013. AVMA Guidelines for the Euthanasia of Animals. American Veterinary Medical Association: Schaumburg, IL. Accessed June 2016. <https:// www.avma.org/KB/Policies/Documents/euthanasia.pdf

Baer, C. K., editor. 2006. Guidelines on Euthanasia of Nondomestic Animals. American Association of Zoo Veterinarians (AAZV): Yulee, FL.

Brown, C. 2010. Fish and Amphibian Euthanasia. CARE306.01. Cornell Center for Animal Resources and Education: Ithaca, NY. Accessed June 2016. <https://www.research.cornell.edu/ care/documents/ACUPs/ACUP306.pdf>.

Farm Animal Welfare Council. 2009. Five Freedoms. Farm Animal Welfare Council: London England. Accessed June 2016. <http://webarchive.nationalarchives.gov.uk/20121007104210/http:/www. fawc.org.uk/freedoms.htm>.

Harrison, G. J., and T. Lightfoot. 2005. Clinical Avian Medicine. Spix: Palm Beach, FL.

Hellebrekers, L., V. Baumans, A. P. Bertens, and W. Hartman. 1990. On the Use of T-61 for Euthanasia of Domestic and Laboratory Animals: An Ethical Evaluation. Laboratory Animals. 24(3): 200-204. 
Hickman, D. L., S. D. Fitz, C. S. Bernable, I. F. Caliman, M. M. Haulcomb, L. M. Federici, A. Shekhar, and P. L. Johnson. 2016. Evaluation of Low Versus High Volume per Minute Displacement $\mathrm{CO}_{2}$ Methods of Euthanasia in the Induction and Duration of Panic-Associated Behavior and Physiology. Animals. 6(8): 45.

Leach, M. C., V. A. Bowell, T. F. Allan, and D. B. Morton. 2004. Measurement of Aversion to Determine Humane Methods of Anesthesia and Euthanasia. Animal Welfare. 13: 77-86.

Lillywhite, H. B., R. Shine, E. Jacobson, D. F. Denardo, M. S. Gordon, C. A. Navas, T. Wang, R. S. Seymour, K. B. Storey, H. Heatwole, D. Heard, B. Brattstrom, and G. M. Burghardt. 2017. Anesthesia and Euthanasia of Amphibians and Reptiles Used in Scientific Research: Should Hypothermia and Freezing Be Prohibited? BioScience. 67: 53-61.

Mader, D. R. 2006. Euthanasia. In Reptile Medicine and Surgery, 2nd edition, Saunders Elsevier: St. Louis, MO.

Makowska, J. I., L. Vickers, J. Mancell, and D. M. Weary. 2009. Evaluating Methods of Gas Euthanasia for Laboratory Mice. Applied Animal Behaviour Science. 121: 230-235.

Meyer, R. E., and W. E. M. Morrow. 2006. Physiology of Euthanasia. Pp. 6-8 in Guidelines on Euthanasia of Nondomestic Animals (C. K. Baer, editor). American Association of Zoo Veterinarians (AAZV): Yulee, FL.

Miller, E. A., editor. 2012. Minimum Standards for Wildlife Rehabilitation, 4th edition. National Wildlife Rehabilitators Association: St. Cloud, $\mathrm{MN}$; International Wildlife Rehabilitation Council: Eugene, OR.

Niel, L., and D. M. Weary. 2006. Behavioural

Responses of Rats to Gradual-fill Carbon Dioxide Euthanasia and Reduced Oxygen Concentrations. Applied Animal Behaviour Science. 100: 295-308.

Paul-Murphy, J. R., A. Engilis Jr., P. J. Pascoe, D. C. Williams, K. A. Gustavsen, T. L. Drazenovich, M. K. Keel, T. M. Polley, and I. E. Engilis. 2017. Comparison of Intraosseous Pentobarbital Administraion and Thoracic Compression for Euthanasia of Anesthetized Sparrows (Passer domesticus) and Starlings (Sturnus vulgaris). American Journal of Veterinary Research. 78(8): 887-899.

Rollin, B. E. 2009. Ethics and Euthanasia. Canadian Veterinary Journal. 50(10): 1081-1086.
Shine, R., J. Amiel, A. J. Munn, M. Stewart, A. L. Vyssotski, and J. A. Lesku. 2015. Is "Cooling then Freezing" a Humane Way to Kill Amphibians and Reptiles? Biology Open. Bio-012179.

Sikes, R. S., W. L. Gannon, and the Animal Care and Use Committee of the American Society of Mammologists. 2011. Guidelines of the American Society of Mammalogists for the Use of Wild Mammals in Research. Journal of Mammology. 92(1): 235-253.

Thurmon, J. C., W. J. Tranquilli, and G. J. Benson, editors. 1996. Lumb and Jones' Veterinary Anesthesia, 3rd edition. Pp. 11-16. Lippincott Williams and Wilkins: Baltimore, MD.

Tidemann, C. R., and D. H. King. 2009. Practicality and Humaneness of Euthanasia of Pest Birds with Compressed Carbon Dioxide $\left(\mathrm{CO}_{2}\right)$ and Carbon Monoxide (CO) from Petrol Engine Exhaust. Wildlife Research 36: 522-527.

Torreilles, S. L., D. E. McClure, and S. L. Green. 2009. Evaluation and Refinement of Euthanasia Methods for Xenopus laevis. Journal of the American Association for Laboratory Animal Science. 48 (5): 512-516.

Woodbury, M. 2014. Euthanasia. In Zoo Animal and Wildlife Immobilization and Anesthesia, 2nd edition (G. West, D. Heard, and N. Caulkett, editors). Wiley-Blackwell: Ames, IA. (Nㅐㄱ

Copyright (C) Wildlife Center of Virginia. Reprinted with permission of the Wildlife Center of Virginia. 